\title{
Variation in Morphological and Biochemical Characters of Various Forage Sorghum Genotypes (Sorghum bicolor L. Moench)
}

\author{
Pinki* and S.S. Siwach \\ College of Agriculture, Department of Genetics and Plant Breeding, CCS Haryana \\ Agricultural University, Hisar-125004, Haryana, India \\ *Corresponding author
}

\section{A B S T R A C T}

\begin{tabular}{|l|}
\hline K e y w o r d s \\
$\begin{array}{l}\text { Sorghum, Morphological } \\
\text { and quality traits, } \\
\text { Analysis of variance, } \\
\text { Mean }\end{array}$ \\
\hline Article Info \\
\hline $\begin{array}{l}\text { Accepted: } \\
\text { 04 May 2018 } \\
\text { Available Online: } \\
\text { 10 June 2018 }\end{array}$ \\
\hline
\end{tabular}

\section{Introduction}

Sorghum [Sorghum bicolor (L.) Moench] is one of the five top cereal crops in the world. Sorghum was also grown in India and Assyria as early as $700 \mathrm{BC}$. Sorghum has a significant role in livestock production, particularly in tropical zone where feed stuffs could not meet animal requirements due to many factors such as poor soil fertility and drought (Pholsen and Suksri, 2007).

Although India is the highest milk producer country yet per capita milk production is very low due to the huge deficit in the availability of feed stuffs. This is also to note that area under forage production has not increased considerably in the last few decades and our natural grazing lands and pastures are fast degrading and decreasing. Hence, efforts should be directed to intensify forage production per unit area per unit time, which can be achieved through improved high yielding varieties and better management practices.

The greater utilization of the genetic diversity in developing sustainable solutions to basic crop constraints or enhancing the productivity will be critical in future. However, for effective utilization of the germplasm collections it is necessary to evaluate and characterize them (Reddy et al., 2006). Therefore, the present investigation was undertaken in forage sorghum with the objective to assess genetic diversity in 
sorghum accessions on the basis of fodder yield and quality.

\section{Materials and Methods}

Twenty five forage sorghum genotypes Bmr1, Bmr-2, Bmr-3, Bmr-4, Bmr-5, COFS 29, HJ 513, HC 260, IS 2205, IS 2389, DUGGI, CSV 21F, S 490, S 437, S 651, G 46, SSG 593, HC 136, HC 171, HJ 541, PC 5, PC 7, PC 8, ICSV 700, HC 308 were used in present study.

The material was collected from Forage Section, Department of Genetics and Plant Breeding CCSHAU, Hisar and sowing was done on July 4, 2015.

The field experiment was conducted at Research Area of Forage Section, Department of Genetics and Plant Breeding, CCS Haryana Agricultural University, Hisar.

Observations were recorded on 31 quantitative variables. The data for different characters were statistically analysed as described by Panse and Sukhatme (1967).

\section{Results and Discussion}

The mean performance and range of different genotypes for different traits have been presented in Table $2 \mathrm{a}$ and $\mathrm{b}$ brief description of the result is given here under:

Natural height of plant up to the base of flag leaf

The range for plant height varied from 88.0$233.3 \mathrm{~cm}$.

Genotype CSV 21F (233.3 cm) showed significantly higher mean followed by SSG 59-3 (211.4), S 651 (208.8) and S 490 (207.1). Number of tillers/plant
Genotype COFS 29 (5.8) had maximum no. of tillers and HC 171 (1.0) had minimum.

\section{Number of leaves/plant}

The number of leaves/plant ranged from 10.630.4. Genotype PC 5 (30.4) had maximum number of leaves/plant followed by HC 308 (25.4).

\section{Stem girth $(\mathbf{m m})$}

Genotype S 490 (12.3 mm), HJ 513(12 mm) showed significant higher stem diameter than over all mean $(8.7 \mathrm{~mm})$. Minimum stem diameter was recorded for genotype COFS 29 $(5.8 \mathrm{~mm})$ while maximum stem diameter was recorded for genotype $S 490(12.3 \mathrm{~mm})$.

\section{Anther length (mm)}

Maximum anther length $(3.3 \mathrm{~mm})$ was observed in genotype SSG 59-3 and minimum $(2.0 \mathrm{~mm})$ in S 651, BMR-5 and COFS29.

\section{Stigma length (mm)}

Maximum stigma length was $2.5 \mathrm{~mm}$ which was recorded in genotype CSV $21 \mathrm{~F}$ followed by $\mathrm{G} 46$ against a minimum of $1.0 \mathrm{~mm}$ was recorded in Duggi.

\section{Time of panicle emergence (no. of days)}

For this trait, genotypes COFS 29 (105 days) and BMR-1 and BMR-4 showed significantly higher than over all mean (79.4 days).

\section{Green fodder yield/plant}

Highest green fodder yield/plant was recorded from CSV 21F (266.6 gm) followed by S 490 (256.5 gm) whereas, minimum from HC 171 (77.7 gm) and overall mean was $161.1 \mathrm{gm}$.

\section{Dry fodder yield/plant}


Maximum dry fodder yield/plant 93.3 gm was recorded from genotype CSV $21 \mathrm{~F}$ while, minimum from $\mathrm{HC} 171$ (27.3 gm) with mean $53.6 \mathrm{gm}$.

\section{Neck of panicle visible above sheath}

For this trait genotypes, SSG 59-3 $(20.6 \mathrm{~cm})$, HJ $541(19.0 \mathrm{~cm})$, HJ $513(17.6 \mathrm{~cm})$ and G 46 $(18.0 \mathrm{~cm})$ were observed to have high neck of panicle visible above sheath.

The range varied from $3.5 \mathrm{~cm}$ in duggi to 20.6 $\mathrm{cm}$ in SSG 59-3.

\section{Panicle length of branches}

For this trait genotypes ICSV $700(14.0 \mathrm{~cm})$, HJ $541(13.3 \mathrm{~cm})$, SSG 59-3 $(13.0 \mathrm{~cm}), \mathrm{S} 437$ $(12.3 \mathrm{~cm})$, showed significantly higher mean.

The range was varied from $4.1 \mathrm{~cm}$ (BMR-3) to $14.0 \mathrm{~cm}$ (ICSV 700).

\section{Lignin (\%)}

The minimum lignin was $2.6 \%$ observed in BMR-3 and maximum $5.8 \%$ in IS 2389 with mean $3.9 \%$.

\section{In vitro Dry Matter Digestibility (IVDMD \%)}

The IVDMD of different genotypes varied from 59.4\% (PC 8) to $68.2 \%$ (BMR-3) with mean $63 \%$.

\section{Crude protein (\%)}

The crude protein was maximum in BMR-2, ICSV700 and HC 171 (9.3\%) and minimum in BMR-5 $(8.2 \%)$. The overall mean was $8.9 \%$.

\section{Neutral detergent fiber (NDF \%)}

It varied from $61.2 \%$ (PC 7) to $75.9 \%$ (BMR-
4) with overall mean $68 \%$.

\section{Acid detergent fiber (ADF \%)}

The range for ADF was $36.1 \%$ (BMR-1) to $56.3 \%$ (COFS 29) with mean $43.6 \%$.

\section{Digestible dry matter (DDM q/ha)}

DDM varied from $26.6 \mathrm{q} / \mathrm{ha}$ (HC 171) to 94.4 q/ha (CSV 21F) with mean 52.7 q/ha.

\section{Protein yield $(\mathrm{q} / \mathrm{ha})$}

Protein yield varied from $3.7 \mathrm{q} / \mathrm{ha}$ (HC 171) to $13.0 \mathrm{q} / \mathrm{ha}$ (CSV 21F) with mean $7.4 \mathrm{q} / \mathrm{ha}$. The mean squares were observed to be highly significant for all the 18 characters studied (Table $3 \mathrm{a}$ and $\mathrm{b}$ ). This indicated prevalence of enough genetic variability in the material under study for selection and improvement of genotypes. This also indicated its suitability for further statistical analysis for all the character studied.

In order to get enhanced performance of animals, the quality of fodder being fed to them is of utmost importance. The main quality attributes in forage sorghum are protein, IVDMD, NDF, ADF. Out of these protein, IVDMD are most important.

Like other straws, the nutritive value of sorghum fodder is also low due to presence of high content of above mentioned cell wall constituents as well as lignin and low content of protein and minerals. Crude protein (CP) content is often considered as a good determinant of quality. Crude protein is commonly used measure of feed quality. Good quality forage generally will have higher protein content.

The major goal in breeding programme is to improve crude protein more than $9 \%$. IVDMD (in vitro dry matter digestibility) is a measure of plant quality index (Kumar and Shib, 2003) (Table 1). 
Table.1 Morphological characters as per DUS parameters

\begin{tabular}{|c|c|c|c|}
\hline & Parameters & States & Stage of observation \\
\hline 1.1 & $\begin{array}{c}\text { Seedling : Anthocyanin colouration } \\
\text { of coleoptiles }\end{array}$ & $\begin{array}{l}\text { Yellow green } \\
\text { Greyed purple }\end{array}$ & $\begin{array}{c}\text { Seedling } \\
7-8 \text { days after sowing }\end{array}$ \\
\hline 1.2 & $\begin{array}{c}\text { Leaf sheath : Anthocyanin } \\
\text { colouration }\end{array}$ & $\begin{array}{l}\text { Yellow green } \\
\text { Greyed purple }\end{array}$ & $5^{\text {th }}$ leaf \\
\hline 1.3 & $\begin{array}{c}\text { Leaf : Midrib colour } \\
\left(5^{\text {th }} \text { fully developed leaf }\right)\end{array}$ & $\begin{array}{l}\text { White } \\
\text { Yellow green } \\
\text { Greyed yellow } \\
\text { Greyed purple }\end{array}$ & $5^{\text {th }}$ leaf \\
\hline 1.4 & $\begin{array}{c}\text { Plant : Time of panicle emergence } \\
\text { (50\% of the plants with } 50 \% \\
\text { anthesis) }\end{array}$ & $\begin{array}{l}\text { Very early ( }<56 \text { days }) \\
\text { Early (56-65 days) } \\
\text { Medium }(66-75 \text { days }) \\
\text { Late }(76-85 \text { days }) \\
\text { Very late }(>85 \text { days })\end{array}$ & Panicle emergence \\
\hline 1.5 & $\begin{array}{l}\text { Plant : Natural height of plant upto } \\
\text { base of flag leaf }\end{array}$ & $\begin{array}{l}\text { Very short }(<76 \mathrm{~cm}) \\
\text { Short }(76-150 \mathrm{~cm}) \\
\text { Medium }(151-225 \mathrm{~cm}) \\
\text { Tall }(226-300 \mathrm{~cm}) \\
\text { Very tall }(>300 \mathrm{~cm})\end{array}$ & Panicle emergence \\
\hline 1.6 & $\begin{array}{l}\text { Flag leaf : Yellow colouration of } \\
\text { midrib }\end{array}$ & $\begin{array}{l}\text { Absent } \\
\text { Present }\end{array}$ & Panicle emergence \\
\hline 1.7 & Lemma : Arista formation & $\begin{array}{l}\text { Absent } \\
\text { Present }\end{array}$ & Flowering \\
\hline 1.8 & Stigma : Anthocyanin colouration & $\begin{array}{l}\text { Absent } \\
\text { Present }\end{array}$ & $\begin{array}{l}\text { Upper portion of the panicle at } \\
\text { the end of flowering }\end{array}$ \\
\hline 1.9 & Stigma : Yellow colouration & $\begin{array}{l}\text { Absent } \\
\text { Present }\end{array}$ & Flowering \\
\hline 1.10 & Stigma : Length & $\begin{array}{l}\text { Short }(<1 \mathrm{~mm}) \\
\text { Medium }(1-2 \mathrm{~mm}) \\
\text { Long }(>2 \mathrm{~mm})\end{array}$ & Flowering \\
\hline 1.11 & $\begin{array}{l}\text { Flower with pedicel : Length of } \\
\text { flower }\end{array}$ & $\begin{array}{l}\text { Very short } \\
\text { Short } \\
\text { Medium } \\
\text { Long } \\
\text { Very long }\end{array}$ & Flowering \\
\hline 1.12 & Anther : Length & $\begin{array}{l}\text { Short }(<3 \mathrm{~mm}) \\
\text { Medium }(3-4 \mathrm{~mm}) \\
\text { Long }(>4 \mathrm{~mm})\end{array}$ & Flowering \\
\hline 1.13 & Anther : Colour of dry anther & $\begin{array}{l}\text { Yellow orange } \\
\text { Orange } \\
\text { Orange red } \\
\text { Grayed orange }\end{array}$ & End of flowering Flowering \\
\hline 1.14 & Number of leaves per plant & $\begin{array}{l}\text { Less }(<15) \\
\text { Medium }(15-40) \\
\text { More }(>40)\end{array}$ & Physiological maturity \\
\hline 1.15 & Stem girth $(\mathrm{cm})$ & $\begin{array}{c}\text { Small }(<2 \mathrm{~cm}) \\
\text { Medium }(2-4 \mathrm{~cm}) \\
\text { Large }(>4 \mathrm{~cm})\end{array}$ & Physiological maturity \\
\hline 1.16 & Number of tillers per plant & $\begin{array}{l}\text { Less }(<2) \\
\text { Medium }(2-5) \\
\text { More }(>5)\end{array}$ & Physiological maturity \\
\hline 1.17 & $\begin{array}{l}\text { Panicle : Length of branches } \\
\text { (middle third of panicle) }\end{array}$ & $\begin{array}{c}\text { Short }(<5.1 \mathrm{~cm}) \\
\text { Medium }(5.1-10 \mathrm{~cm}) \\
\text { Long }(10.1-15 \mathrm{~cm}) \\
\text { Very long }(>15 \mathrm{~cm})\end{array}$ & Physiological maturity \\
\hline
\end{tabular}




\begin{tabular}{|c|c|c|c|}
\hline 1.18 & $\begin{array}{l}\text { Panicle : Density at maturity } \\
\text { (ear head compactness) }\end{array}$ & $\begin{array}{l}\text { Very loose } \\
\text { Loose } \\
\text { Semi loose } \\
\text { Semi compact } \\
\text { Compact }\end{array}$ & Physiological maturity \\
\hline 1.19 & Panicle : Shape & $\begin{array}{c}\text { Reversed pyramid } \\
\text { Panicle broader in upper part } \\
\text { Symmetric } \\
\text { Panicle broader in lower part } \\
\text { Pyramidal }\end{array}$ & Physiological maturity \\
\hline 1.20 & $\begin{array}{l}\text { Neck of panicle : Visible length } \\
\text { above sheath }\end{array}$ & $\begin{array}{c}\text { Absent or very short }(<5.1 \mathrm{~cm}) \\
\text { Short }(5.1-10 \mathrm{~cm}) \\
\text { Medium }(10.1-15 \mathrm{~cm}) \\
\text { Long }(15.1-20 \mathrm{~cm}) \\
\text { very long }(>20 \mathrm{~cm})\end{array}$ & Physiological maturity \\
\hline 1.21 & Glume : Length & $\begin{array}{l}\text { Very short ( } 25 \% \text { of grain covered) } \\
\text { Short ( } 50 \% \text { of grain covered) } \\
\text { Medium ( } 75 \% \text { of grain covered) } \\
\text { Long ( } 100 \% \text { of grain covered) } \\
\text { Very long (longer than the grain) }\end{array}$ & Physiological maturity \\
\hline 1.22 & Grain : Threshability & $\begin{array}{l}\text { Freely threshable } \\
\text { (<11\% unthreshed grain) } \\
\text { Partly thresahble } \\
\text { (11 }-50 \% \text { unthreshed grain) } \\
\text { Difficult to thresh } \\
\text { (>50\% unthreshed grain) }\end{array}$ & Maturity \\
\hline 1.23 & Caryopsis : Colour after threshing & $\begin{array}{l}\text { White RHS } 155 \\
\text { Greyed white RHS } 156 \\
\text { Yellow white RHS } 158 \\
\text { Yellow orange RHS } 14-20 \\
\text { Greyed orange RHS } 200\end{array}$ & After threshing \\
\hline 1.24 & Grain : Shape (in dorsal view) & $\begin{array}{l}\text { Narrow elliptic } \\
\text { Elliptic } \\
\text { Circular }\end{array}$ & After threshing \\
\hline 1.25 & Grain : Shape in profile view & $\begin{array}{l}\text { Narrow elliptic } \\
\text { Elliptic } \\
\text { Circular }\end{array}$ & After threshing \\
\hline 1.26 & Grain : Size of mark of germ & $\begin{array}{l}\text { Very small } \\
\text { Small } \\
\text { Medium } \\
\text { Large } \\
\text { Very large }\end{array}$ & After threshing \\
\hline 1.27 & $\begin{array}{l}\text { Grain : Texture of endosperm } \\
\quad \text { (in longitudinal section) }\end{array}$ & $\begin{array}{c}\text { Fully vitreous ( } 100 \% \text { corneous }) \\
3 / 4 \text { vitreous }(75 \% \text { corneous }) \\
\text { Half vitreous }(50 \% \text { corneous }) \\
3 / 4 \text { farinaceous }(25 \% \text { corneous }) \\
\text { Fully farinaceous }(0 \% \text { corneous })\end{array}$ & After threshing \\
\hline 1.28 & Grain : Colour of vitreous albumen & $\begin{array}{c}\text { Greyed yellow RHS 160-162 } \\
\text { Greyed orange RHS } 166 \\
\text { Greyed purple RHS N } 187\end{array}$ & After threshing \\
\hline 1.29 & Grain : Luster & $\begin{array}{l}\text { Non-lustrous } \\
\text { Lustrous }\end{array}$ & After threshing \\
\hline 1.30 & Green fodder yield/plant & & After harvesting \\
\hline 1.31 & Dry fodder yield/plant & & After harvesting \\
\hline
\end{tabular}


Table.2 (a) Mean performance and range of different morphological traits

\begin{tabular}{|c|c|c|c|c|c|c|c|c|c|}
\hline Genotypes & $\begin{array}{l}\text { Natural } \\
\text { height of } \\
\text { plant upto } \\
\text { base of flag } \\
\text { leaf }(\mathrm{cm})\end{array}$ & $\begin{array}{l}\text { No. of } \\
\text { tillers } \\
\text { /plant }\end{array}$ & $\begin{array}{l}\text { No. of } \\
\text { leaves } \\
\text { /plant }\end{array}$ & $\begin{array}{l}\text { Stem } \\
\text { girth } \\
(\mathrm{mm})\end{array}$ & $\begin{array}{l}\text { Anther } \\
\text { length } \\
(\mathrm{mm})\end{array}$ & $\begin{array}{l}\text { Stigma } \\
\text { length } \\
(\mathrm{mm})\end{array}$ & $\begin{array}{l}\text { Time of } \\
\text { panicle } \\
\text { emergence } \\
\text { (no. of } \\
\text { days) }\end{array}$ & $\begin{array}{l}\text { Green } \\
\text { fodder } \\
\text { yield/plant } \\
\text { (g) }\end{array}$ & $\begin{array}{l}\text { Dry fodder } \\
\text { yield/plant } \\
\text { (g) }\end{array}$ \\
\hline BMR-1 & 94.0 & 1.8 & 16.5 & 10.9 & 2.3 & 2.3 & 102.0 & 150.5 & 51.9 \\
\hline BMR-2 & 137.9 & 1.3 & 17.3 & 9.4 & 2.8 & 2.3 & 62.6 & 152.7 & 53.2 \\
\hline BMR-3 & 88.0 & 1.4 & 16.5 & 9.8 & 2.1 & 2.3 & 73.0 & 127.7 & 48.6 \\
\hline BMR-4 & 109.2 & 1.5 & 14.0 & 8.2 & 3.1 & 2.0 & 101.0 & 165.5 & 56.2 \\
\hline BMR-5 & 178.3 & 1.1 & 10.6 & 9.9 & 2.0 & 2.3 & 79.3 & 152.2 & 46.9 \\
\hline COFS 29 & 173.3 & 5.8 & 19.3 & 5.8 & 2.0 & 2.4 & 105.0 & 90.5 & 31.6 \\
\hline HJ 513 & 194.6 & 1.4 & 19.7 & 12.0 & 2.5 & 1.6 & 78.6 & 183.3 & 60.0 \\
\hline HC 260 & 202.7 & 1.7 & 16.5 & 8.9 & 3.1 & 1.6 & 60.3 & 147.2 & 46.5 \\
\hline IS 2205 & 201.5 & 1.8 & 20.2 & 8.6 & 3.2 & 2.2 & 59.0 & 182.7 & 58.7 \\
\hline IS 2389 & 179.5 & 2.6 & 24.9 & 8.9 & 2.1 & 1.9 & 67.6 & 197.8 & 65.5 \\
\hline DUGGI & 147.9 & 1.9 & 20.1 & 6.4 & 2.3 & 1.0 & 68.0 & 126.6 & 48.0 \\
\hline CSV 21F & 233.3 & 1.7 & 19.0 & 8.5 & 3.3 & 2.5 & 80.6 & 266.6 & 93.3 \\
\hline S 490 & 207.1 & 1.5 & 16.4 & 12.3 & 3.2 & 1.8 & 90.3 & 256.5 & 93.3 \\
\hline S 437 & 180.7 & 1.7 & 16.3 & 9.1 & 3.1 & 2.3 & 69.0 & 173.1 & 58.0 \\
\hline S651 & 208.8 & 2.2 & 16.3 & 11.7 & 2.0 & 2.0 & 89.3 & 186.9 & 61.5 \\
\hline G 46 & 174.0 & 2.2 & 20.0 & 7.4 & 2.1 & 2.5 & 60.6 & 120.0 & 38.5 \\
\hline SSG 59-3 & 211.4 & 2.3 & 15.4 & 7.2 & 3.3 & 2.3 & 71.6 & 103.3 & 38.2 \\
\hline HC 136 & 151.3 & 1.9 & 20.7 & 5.9 & 2.3 & 1.9 & 91.3 & 152.2 & 49.9 \\
\hline HC 171 & 186.0 & 1.0 & 12.0 & 7.5 & 3.1 & 2.5 & 91.6 & 77.7 & 27.2 \\
\hline HJ 541 & 180.4 & 1.5 & 19.5 & 8.2 & 3.2 & 2.4 & 83.0 & 209.7 & 48.4 \\
\hline PC 5 & 151.1 & 2.6 & 30.4 & 7.6 & 2.0 & 1.4 & 77.6 & 182.8 & 61.0 \\
\hline PC 7 & 172.7 & 1.8 & 18.1 & 8.0 & 3.2 & 2.4 & 82.0 & 129.9 & 41.8 \\
\hline PC 8 & 148.5 & 1.7 & 18.8 & 9.3 & 2.4 & 1.8 & 95.6 & 181.6 & 64.0 \\
\hline ICSV 700 & 176.9 & 2.2 & 17.5 & 8.4 & 2.1 & 1.3 & 62.0 & 150.5 & 58.7 \\
\hline HC 308 & 104.4 & 2.2 & 25.4 & 7.6 & 2.5 & 1.6 & 89.3 & 159.5 & 38.9 \\
\hline Mean & 167.7 & 1.9 & 18.4 & 8.7 & 2.6 & 2.0 & 79.4 & 161.1 & 53.6 \\
\hline Range & $88.0-233.3$ & $1.0-5.8$ & $\begin{array}{l}10.6- \\
30.4\end{array}$ & $\begin{array}{l}5.8- \\
12.3\end{array}$ & $2.0-3.3$ & $1.0-2.5$ & $59-105$ & $77.7-266.6$ & $27.2-93.3$ \\
\hline C.V \% & 12.9 & 17.5 & 13.7 & 5.3 & 10.7 & 7.8 & 3.4 & 11.8 & 5.1 \\
\hline
\end{tabular}


Table.2 (b) Mean performance and range of different morphological and quality traits

\begin{tabular}{|c|c|c|c|c|c|c|c|c|c|}
\hline Genotypes & $\begin{array}{l}\text { Neck of } \\
\text { panicle } \\
\text { visible above } \\
\text { the sheath } \\
(\mathrm{cm})\end{array}$ & $\begin{array}{l}\text { Panicle :length } \\
\text { of } \\
\text { branches }(\mathrm{cm})\end{array}$ & $\begin{array}{l}\text { Lignin } \\
(\%)\end{array}$ & $\begin{array}{l}\text { IVDMD } \\
(\%)\end{array}$ & $\begin{array}{l}\text { Crude } \\
\text { protein } \\
(\%)\end{array}$ & $\begin{array}{l}\text { NDF } \\
(\%)\end{array}$ & $\begin{array}{l}\text { ADF } \\
(\%)\end{array}$ & $\begin{array}{l}\text { DDM } \\
\text { (q/ha) }\end{array}$ & $\begin{array}{l}\text { Protein } \\
\text { yield } \\
\text { (q/ha) }\end{array}$ \\
\hline BMR-1 & 3.5 & 5.1 & 3.1 & 60.4 & 8.3 & 65.5 & 36.5 & 48.8 & 6.4 \\
\hline BMR-2 & 7.0 & 4.6 & 3.0 & 60.4 & 9.3 & 70.1 & 36.1 & 48.0 & 7.2 \\
\hline$\overline{\text { BMR-3 }}$ & 5.0 & 4.1 & 2.6 & 68.2 & 9.1 & 65.6 & 45.8 & 38.5 & 6.0 \\
\hline BMR-4 & 6.1 & 7.0 & 3.0 & 61.6 & 8.8 & 75.9 & 44.6 & 54.8 & 7.5 \\
\hline BMR-5 & 6.5 & 6.0 & 4.3 & 60.2 & 8.2 & 70.3 & 46.2 & 44.0 & 5.9 \\
\hline COFS 29 & 12.8 & 9.3 & 5.7 & 60.2 & 8.4 & 68.0 & 56.3 & 28.3 & 3.9 \\
\hline HJ 513 & 17.6 & 7.0 & 3.3 & 65.5 & 9.0 & 69.3 & 37.4 & 59.8 & 8.5 \\
\hline HC 260 & 11.8 & 12.0 & 3.7 & 60.2 & 8.6 & 67.3 & 36.4 & 52.3 & 5.8 \\
\hline IS 2205 & 11.1 & 8.3 & 3.5 & 64.2 & 8.6 & 65.3 & 43.3 & 58.4 & 7.6 \\
\hline IS 2389 & 6.5 & 7.0 & 5.8 & 65.2 & 9.0 & 67.6 & 48.4 & 63.6 & 8.8 \\
\hline DUGGI & 3.5 & 10.0 & 3.8 & 65.7 & 9.0 & 67.5 & 43.5 & 44.9 & 6.2 \\
\hline CSV 21F & 8.0 & 7.0 & 3.7 & 68.2 & 9.4 & 67.6 & 43.0 & 94.4 & 13.0 \\
\hline S 490 & 7.0 & 7.0 & 3.4 & 68.1 & 9.0 & 64.3 & 42.3 & 90.4 & 11.9 \\
\hline S 437 & 4.8 & 12.3 & 3.5 & 60.8 & 9.2 & 74.2 & 42.3 & 52.9 & 8.0 \\
\hline S 651 & 10.6 & 9.1 & 5.4 & 67.2 & 8.4 & 67.9 & 56.3 & 64.0 & 8.0 \\
\hline G 46 & 18.0 & 12.3 & 3.3 & 64.0 & 9.0 & 64.1 & 41.7 & 38.8 & 5.3 \\
\hline SSG 59-3 & 20.6 & 13.0 & 4.5 & 60.0 & 9.2 & 66.2 & 42.6 & 32.1 & 4.6 \\
\hline HC 136 & 6.5 & 5.3 & 5.4 & 62.3 & 9.1 & 68.1 & 39.6 & 49.1 & 6.6 \\
\hline HC 171 & 11.1 & 6.3 & 3.5 & 66.1 & 9.3 & 71.6 & 43.5 & 26.6 & 3.7 \\
\hline HJ 541 & 19.0 & 13.3 & 3.6 & 65.1 & 9.1 & 68.6 & 44.2 & 64.1 & 8.4 \\
\hline PC 5 & 8.5 & 8.0 & 3.2 & 66.2 & 8.9 & 68.6 & 44.6 & 62.7 & 8.6 \\
\hline PC 7 & 6.8 & 8.3 & 3.2 & 62.3 & 8.7 & 61.2 & 43.3 & 40.6 & 5.5 \\
\hline PC 8 & 5.0 & 8.0 & 4.1 & 59.4 & 8.9 & 68.3 & 43.2 & 56.0 & 8.6 \\
\hline ICSV 700 & 16.6 & 14.0 & 4.7 & 62.0 & 9.3 & 68.5 & 46.1 & 68.0 & 9.9 \\
\hline $\begin{array}{l}\text { HC } 308 \\
\end{array}$ & 4.5 & 8.3 & 3.5 & 61.8 & 9.0 & 68.1 & 42.2 & 36.1 & 9.5 \\
\hline Mean & 9.5 & 8.5 & 3.9 & 63.0 & 8.9 & 68.0 & 43.6 & 52.7 & 7.4 \\
\hline Range & $3.5-20.6$ & $4.1-14.0$ & $2.6-5.8$ & $59.4-68.2$ & $8.2-9.3$ & $\begin{array}{l}61.2- \\
75.9\end{array}$ & $\begin{array}{l}36.1- \\
56.3\end{array}$ & $\begin{array}{l}26.6- \\
94.4\end{array}$ & $3.7-13.0$ \\
\hline C.V & 10.0 & 9.4 & 5.3 & 0.8 & 2.1 & 0.4 & 0.5 & 7.5 & 6.5 \\
\hline
\end{tabular}

Table.3 (a) Analysis of variance for different morphological characters

\begin{tabular}{|c|c|c|c|c|c|c|c|c|c|c|}
\hline $\begin{array}{l}\text { Source of } \\
\text { variation }\end{array}$ & $\begin{array}{l}\text { D. } \\
\text { F }\end{array}$ & $\begin{array}{l}\text { Green } \\
\text { fodder } \\
\text { yield/plant } \\
\text { (gm) }\end{array}$ & $\begin{array}{l}\text { Natural } \\
\text { height of } \\
\text { plant } \\
\text { upto base } \\
\text { of flag } \\
\text { leaf }(\mathrm{cm})\end{array}$ & $\begin{array}{l}\text { No. of } \\
\text { leaves/pl } \\
\text { ant }\end{array}$ & $\begin{array}{l}\text { Stem } \\
\text { girth } \\
(\mathbf{m m})\end{array}$ & $\begin{array}{l}\text { No. of } \\
\text { tillers/plant }\end{array}$ & $\begin{array}{l}\text { Dry fodder } \\
\text { yield/plant } \\
\text { (gm) }\end{array}$ & $\begin{array}{l}\text { Time of } \\
\text { panicle } \\
\text { emergen } \\
\text { ce (no. } \\
\text { of days) }\end{array}$ & $\begin{array}{l}\text { Stigma } \\
\text { Length } \\
(\mathrm{mm})\end{array}$ & $\begin{array}{l}\text { Anther } \\
\text { Length }(\mathrm{mm})\end{array}$ \\
\hline Replication & 2 & 2291.66 & 503.34 & 88.61 & 0.012 & 3.72 & 8.87 & 53.32 & 0.27 & 0.03 \\
\hline Genotype & 24 & $5939.64 * *$ & $4383.75^{* *}$ & $51.13 * *$ & $8.58 * *$ & $2.46^{* *}$ & $735.46 * *$ & $566.6^{* *}$ & $0.51 * *$ & $0.80 * *$ \\
\hline Error & 48 & 367.07 & 475.21 & 6.47 & 0.21 & 0.12 & 7.54 & 7.65 & 0.02 & 0.08 \\
\hline
\end{tabular}

* Significant at $5 \%$ level; **significant at $1 \%$ level 
Table.3 (b) Analysis of variance for different morphological and quality characters

\begin{tabular}{|l|l|l|l|l|l|l|l|l|l|l|}
\hline Source of variation & D.F & $\begin{array}{l}\text { Panicle } \\
\text { :length of } \\
\text { branches } \\
\text { (cm) }\end{array}$ & $\begin{array}{l}\text { Neck of } \\
\text { panicle } \\
\text { above } \\
\text { sheath } \\
\text { (cm) }\end{array}$ & $\begin{array}{l}\text { Lignin } \\
\text { \% }\end{array}$ & $\begin{array}{l}\text { IVDMD } \\
\text { \% }\end{array}$ & C.P \% & NDF \% & ADF \% & $\begin{array}{l}\text { DDM } \\
\text { (q/ha) }\end{array}$ & $\begin{array}{l}\text { Protein } \\
\text { yield } \\
(\mathbf{q} / \text { ha })\end{array}$ \\
\hline Replication & 2 & 5.96 & 3.62 & 0.03 & 0.05 & 0.007 & 0.10 & 0.005 & 38.19 & 0.05 \\
\hline Genotype & 24 & $24.53 * *$ & $81.14^{* *}$ & $2.36^{* *}$ & $25.96 * *$ & $0.332^{* *}$ & $28.09 * *$ & $73.62 * *$ & $833.05 *$ & $15.10^{* *}$ \\
\hline Error & 48 & 0.65 & 0.91 & 0.04 & 0.26 & 0.03 & 0.09 & 0.06 & 15.63 & 0.24 \\
\hline
\end{tabular}

* Significant at $5 \%$ level; **significant at $1 \%$ level

In present investigation results from variability studies revealed that crude protein and IVDMD varied from 8.2 to $9.4 \%$ and 59.4 to $68.2 \%$, respectively. Findings of present investigation is in close conformation with the findings of Gupta et al., (2002) who revealed that protein and IVDMD varied from 3.01 to 8.75 and 40.40 to $66.16 \%$, respectively. Singh et al., (2010) reported that protein content in single cut (SC) and multicut (MC) genotypes ranged from 5.24 to 10.06 and 4.81 to12.47 per cent, respectively. Similarly, IVDMD ranged from 50.4 to 62.0 and 48.3 to 62.2 per cent, respectively. In present investigation genotypes BMR-2, HC 171 (9.3) and S 437 (9.2) observed high crude protein and BMR-3 (68.2), S 490 (68.1) and S 651 (67.2) observed high IVDMD. Digestible dry matter (DDM) is the portion of the dry matter in a feed that is digested by animal at specific level of feed intake. DDM ranged from $26.6 \mathrm{q} /$ ha to $94.4 \mathrm{q} / \mathrm{ha}$, being maximum in CSV 21F (94.4). The digestibility of sorghum fodder is affected by cell wall constituents' viz., NDF, ADF and lignin. NDF measures total cell wall content of plant. The concentration of NDF in feeds is negatively correlated with energy concentration and lowers the quality of forage. As ADF increases, digestibility and nutrient availability decreases. In our findings NDF, $\mathrm{ADF}$, and lignin ranged from 61.2 to 75.9 , 36.1 to 56.3 and 2.6 to 5.8 per cent, respectively. These results are in conformity with that of Grenier et al., (2001) who reported that $\mathrm{NDF}, \mathrm{ADF}$, and lignin ranged from 57.48 to $70.00,30.85$ to 42.80 and 3.80 to 7.00 per cent, respectively. All BMR genotypes show low amount of lignin in BMR-3, BMR-2, BMR-4, BMR-1 i.e. 2.6, 3.0, 3.0, 3.1 respectively.

\section{References}

Grenier, C. P. J., Bramel-Cox, M. Noirot. K.E. Prasada Rao and Hamon, P. (2001) Assessment of genetic diversity in three subset consitituted from the ICRISAT sorghum collection using random vs non-random sampling procedures. Theor. Appli. Genet, 101, 190-196

Gupta, S.K., Verma, M.L. and Jaiswal, R.S. (2002) Nutrition evaluation of multicut sorghum strains. Indian J. Anim. Nutr, 19(2), 129-134.

Kumar, M.H. and Sahib, K.H. (2003) Genetic studies and correlations of biomass related characters in forage sorghum under abiotic stress conditions. J. Res. Amgrau, 31(3), 35-39.

Panse, V.G. and Sukhatme, P.V. (1967) Statistical methods for agricultural worker, $4^{\text {th }}$ edd. Indian council of Agricultural Research, New Delhi.

Pholsen, S. and Suksri, A. (2007) Effect of phosphorus and potassium on growth, yield and fodder quality of IS 23585 forage sorghum cultivar (Sorghum bicolor (L.) Moench). Pakistan J. Bio. Sci, 10, 1604-1610. 
Reddy, B.V.S., Sharma, H.C., Thakur, R.P., Sorghum hybrid parents (set I). Ramesh, S. (2006) Special issue: Characterization of ICRISAT- bred International Sorghum and Millets Newsletter, 47, 138.

\section{How to cite this article:}

Pinki and Siwach, S.S. 2018. Variation in Morphological and Biochemical Characters of Various Forage Sorghum Genotypes (Sorghum bicolor L. Moench). Int.J.Curr.Microbiol.App.Sci. 7(06): 481-489. doi: https://doi.org/10.20546/ijcmas.2018.706.053 